\title{
RESISTÊNCIA ANTI-HELMÍNTICA EM CAPRINOS NO MUNICÍPIO DE MOSSORÓ, RN
}

\author{
Wesley Adson Costa Coelho, ${ }^{1}$ Sílvia Maria Mendes Ahid, ${ }^{1}$ Luiz da Silva Vieira, ${ }^{2}$ Zuliete Aliona Araújo de \\ Souza Fonseca ${ }^{3}$ E Idalécio Pacífico da Silva ${ }^{4}$ \\ 1. Laboratório de Parasitologia Animal, Universidade Federal Rural do Semiárido, UFERSA, km 47, BR 110, CEP 59625-000. \\ E-mail: wesleyadson@hotmail.com \\ 2. Embrapa Caprinos, km 4, Cx. Postal D10, Sobral, CE \\ 3. Graduando de Medicina Veterinária, UFERSA \\ 4. Mestre em Ciência Animal, UFERSA
}

\section{RESUMO}

O Nordeste brasileiro destaca-se na caprinocultura, onde as helmintoses gastrintestinais estão entre os maiores problemas. Mesmo com drogas anti-helmínticas de amplo espectro, o controle dos parasitos vem sendo prejudicado pela resistência parasitária. $\mathrm{O}$ objetivo deste trabalho foi verificar a sensibilidade dos nematodeos de caprinos à ação dos vermífugos ivermectina $1 \%$ e albendazole $10 \%$, como também determinar o perfil das propriedades com helmintos resistentes aos princípios ativos em questão. O trabalho foi realizado no período de janeiro a novembro de 2008 em trinta criações de caprinos em Mossoró, RN. Em cada propriedade, selecionaram-se 45 animais, divididos em três categorias. Eram vermifugados dez dias pós-tratamento, coletaram-se amostras de fezes. As coordenadas geográficas de todas as propriedades foram obtidas por GPS. Analisaram-se os dados pelo programa estatístico RESO. Das propriedades estudadas, o Haemonchus contortus teve percentual de $90 \%$ resistência ao albendazole em $30,6 \%$ à ivermectina. Este, por sua vez, foi o mais prevalente em todos os grupos tratados. Já o Trichostrongylus sp. foi encontrado em $70 \%$ das propriedades e percentual da população de helmintos resistentes à ivermectina foi de $33,3 \%$, e ao albendazole de $42,8 \%$. A redução na contagem de ovos nas fezes (RCOF) dez dias póstratamento com ivermectina e albendazole variou entre $43 \%$ e $100 \%$ e $29 \%$ e $100 \%$, respectivamente. O perfil das propriedades demonstrou que os anti-helmínticos mais utilizados pertenciam aos grupos dos benzimidazóis $(63,3 \%)$, das avermectinas $(33,3 \%)$ e dos imidazois $(3,3 \%)$.

PALAVRAS-CHAVES: Anti-helmínticos, caprinos, resistência.

\section{ABSTRACT}

\section{ANTHELMINTIC RESISTANCE OF GOATS IN THE CITY OF MOSSORO, RN, BRAZIL}

The Brazilian Northeast area is known for the caprineculture, whose main problem is the gastrointestinal nematodes, and the control of the parasites has been jeopardized by the anthelmintic resistance. The purpose of this study was to verify the sensibility of the goat nematodes to the activity of the substances ivermectin $1 \%$ and albendazole $10 \%$. The work was carried out in 30 goat herds located in the city of Mossoró, Rio Grande do Norte, Brazil, within January and November, 2008. In each property, 45 animals were selected and divided into three groups (one control and two treatments). The geographical coordinates of all the properties were obtained by GPS. The obtained data were analyzed by the statistical program RESO. The animals received the vermifude and 10 days after-treatment feces were collected. In the studied properties, the Haemonchus contortus showed greater prevalence in all the treated groups, $90 \%$ of the helmints population was resistant to albendazole and $36.6 \%$ to ivermectina. Trichostrongylus sp. was found in $70 \%$ of the properties and its percentile of the helmint population resistant to ivermectina and to albendazole was of $33.3 \%$ and $42.8 \%$, respectively. The reduction in the egg counting in the feces (RECF) after 10 days of treatment with ivermectina and albendazole varied within $43 \%$ and $100 \%$, and within $29 \%$ to $100 \%$, respectively. The profile of the properties demonstrated that the anthelmintic most commonly used were from the following groups: benzimidazol (63.3\%), ivermectin (33.3\%), and imidazol (3.3\%).

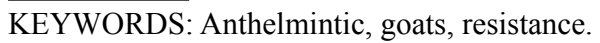




\section{INTRODUÇÃO}

No semiárido brasileiro, o Nordeste tem sido, durante séculos, área de vocação pecuária, especialmente para a exploração dos ruminantes domésticos. Dentre as várias alternativas encontradas para a exploração agropecuária da região, destaca-se a caprinocultura como uma alternativa econômica viável de geração de emprego e renda (SIMPLICIO, 2006), sendo uma das principais atividades econômicas das áreas mais secas do Nordeste (COSTA JÚNIOR et al., 2005).

No estado do Rio Grande do Norte o rebanho está em ascensão. Em 2003, a população de caprino era de 406 mil (IBGE, 2003). Atualmente, o Estado destaca-se na produção de carne e leite (ACOSC, 2006). No entanto, a caprinocultura sofre grandes perdas econômicas, em virtude das verminoses, que, dentre os fatores que as favorecem, estão o manejo, o estado fisiológico, as condições climáticas, a lotação e a nutrição (MATTOS \& CASTRO, 2002).

O parasitismo por nematoides gastrintestinais tem-se constituído em um dos principais fatores limitantes à exploração da caprinocultura, sendo um aspecto importante no manejo sanitário, pelo fato destes animais serem relativamente sensíveis a endoparasitas, principalmente os gastrintestinais. Haemonchus contortus, Trichostrongylus sp., Cooperia sp., Oesophagostomum columbianum, Trichuris sp., Cysticercus sp., e Strongyloides papilosus são responsáveis por significativas perdas econômicas, em decorrência da elevada taxa de mortalidade de animais e atraso no crescimento (COSTA JR. et al., 2005). Na região oeste do Rio Grande do Norte, os helmintos de maior prevalência são os Strongyloides sp., seguidos por Haemonchus sp., Trichostrongylus sp. e, em menor escala, por Oesophagostomum sp. (AHID et al., 2008).

O controle dos parasitos vem sendo realizado através do uso de anti-helmínticos pertencentes a diversos grupos químicos, na maioria das vezes, sem considerar os fatores epidemiológicos predominantes na região, os quais interferem diretamente na população parasitária ambiental e, consequentemente, na infecção do rebanho (VIEIRA \& CAVALCANTE, 1999).

Vários princípios ativos vêm sendo utilizados no tratamento de nematoides de caprino, os quais são classificados em quatro grandes grupos, conforme seu mecanismo de ação, espectro de atividade e eficácia: grupo I - benzimidazóis e pró-benzimidazóis; grupo II- imidazóis e pirimidinas; grupo III - salicilanilidas e substitutos nitrofenólicos; grupo IV - organofosforados (MATTOS \& CASTRO, 2002). Além desses grupos, a partir da década de 1980 foi criada a ivermectina, que atua tanto em ectoparasitos como em endoparasitos. Esta, por sua vez, é derivada de um novo grupo, denominado lactona macrocíclica (avermectinas) (MELO et al., 2003).

A resistência anti-helmíntica (RA) é um fenômeno pelo qual alguns organismos de uma população são capazes de sobreviver após constante utilização de um composto químico (MOLENTO, 2004). A resistência dos nematodeos gastrintestinais de caprinos aos anti-helmínticos foi descrito inicialmente no Texas, Estados Unidos (THEODORIDES et al., 1970), posteriormente, na Nova Zelândia (KETTLE et al., 1983) e França (KERBOUEF \& HUBERT, 1985). No Brasil, a primeira suspeita de nematoides gastrintestinais de caprinos resistentes aos anti-helmínticos foi descrita por VIEIRA (1986) no estado do Ceará, posteriormente foi identificada RA de caprinos em Pernambuco (CHARLES et al., 1989) e na Bahia (BARRETO \& SILVA, 1999).

Segundo PAIVA et al. (2007), o mecanismo de instalação da resistência ocorre pelo uso frequente $\mathrm{e}$ continuado de uma mesma base farmacológica destinada ao controle dos parasitos. De acordo com MELO et. al. (1998), a resistência é agravada pela frequência de tratamentos anti-helmínticos e pela rotação rápida de principio ativo. A utilização de medicamentos de longa persistência e a aquisição de animais contaminados devem ser considerados como causas predisponentes ao aparecimento da RA (MOLENTO, 2004). Acrescenta-se ainda o uso de subdoses, que também pode ser um fator para a formação da RA (VIEIRA \& CAVALCANTE 1999).

A RA pode ser diagnosticada pela utilização de testes específicos, por métodos diretos ou indiretos. No método direto, tem-se o teste in vivo controlado. Nos métodos indiretos, são comumente empregadas duas abordagens na identificação de possibilidade de resistência: as análises in vitro e o teste de redução na contagem de ovos por grama de fezes (FECRT - fecal egg count reduction test) (ALBERTI et al., 2001). Para este último, o diagnóstico será positivo para "resistência" quando uma determinada droga que apresentava redução acima 
de $99 \%$ da carga parasitária apresentar redução menor do que $95 \%$ contra determinado organismo após certo período de tempo (MOLENTO, 2004).

Diferentes esquemas de tratamento são utilizados a fim de reduzir ou eliminar os efeitos adversos do parasitismo. Dos esquemas de controle, o estratégico é o mais utilizado, sendo aplicados tratamentos com um anti-helmíntico de alta eficácia, antes que ocorra um aumento significativo da população de parasitos em épocas do ano predeterminadas. Um dos grandes riscos desse controle refere-se à pequena ou nenhuma população de nematoides em refúgio durante a época seca. Esse fato, aliado à utilização de tratamento anti-helmínticos nesses períodos, possibilita que a resistência anti-helmíntica desenvolva-se rapidamente (SANGSTER, 2001).

Em virtude da importância do assunto e pelo fato de estar diretamente ligado ao fator econômico da região, este trabalho teve por objetivo detectar a ocorrência de resistência aos anti-helmínticos ivermectina $1 \%$ e albendazole $10 \%$ em nematodeos gastrintestinais de caprinos, como também determinar o perfil das propriedades com helmintos resistentes aos princípios ativos em questão, no município de Mossoró, RN.

\section{MATERIAL E MÉTODOS}

Durante o período de janeiro a novembro de 2008 desenvolveu-se o experimento em explorações tradicionais do sistema produtivo de caprinos no município de Mossoró, $\mathrm{RN}$, onde estão concentrados os maiores efetivos do Rio Grande do Norte. A escolha das propriedades obedeceu ao método de amostragem aleatória. As análises laboratoriais foram enviadas e processadas no Laboratório de Parasitologia Animal da Universidade Federal Rural do Semiárido (UFERSA), Mossoró, RN.

Visitaram-se ao total trinta assentamentos rurais, cadastrados junto aos programas assistencialistas do Instituto de Assistência Técnica e Extensão Rural do RN (EMATER-RN) e junto às Associações organizadas de criadores de caprinos e ovinos no Estado, onde foi feita a coleta das fezes e obtiveram-se, por meio de questionários, as informações sobre os métodos antiparasitários usados. Para o experimento, foram utilizados dois grupos de medicamentos pertencentes aos benzimidazóis (albendazole) e lactonas (iver- mectina). Para a seleção e inclusão dos animais no experimento, obedeceu-se aos seguintes critérios: diagnóstico positivo para helmintos gastrintestinais, através da contagem do número de ovos por grama de fezes (OPG); animais que não tenham sido tratados com qualquer tipo de droga anti-helmíntica por um período mínimo de trinta dias.

Por propriedade, selecionaram-se 45 animais da espécie caprina, variando entre a primeira e segunda muda dentária, marcados por meio de brincos e/ou colares, sendo em seguida distribuídos aleatoriamente por rebanho de caprinos em três grupos de quinze animais cada. O grupo I recebeu tratamento com albendazole a $10 \%$, o grupo II recebeu ivermectina e o grupo III foi o controle, não recebendo o tratamento convencional. As doses utilizadas seguiram as recomendadas pelos fabricantes. O grupo I foi tratado com anti-helmíntico à base de albendazole, administrado oralmente, na dose $10 \mathrm{mg} / \mathrm{Kg}$; o grupo II, tratado com anti-helmíntico à base de ivermectina, administrado por via subcutânea, na dose $0,2 \mathrm{mg} / \mathrm{Kg}$.

De cada animal hospedeiro (caprino), naturalmente infectado por nematoides gastrintestinais, foram coletadas amostras fecais diretamente da ampola retal, identificadas em sacos plásticos e mantidas sob refrigeração até o processamento da determinação do número de ovos por grama de fezes e para a obtenção das larvas. As coletas foram feitas no dia do tratamento e dez dias pós-tratamento.

Das amostras coletadas, retiraram-se dois gramas de fezes para a análise quantitativa pela contagem de ovos por grama de fezes (OPG), pela técnica de GORDON \& WHITLOCK (1939). Para recuperação de larvas infectantes, empregou-se a técnica de coprocultura quantitativa, descrita por UENO \& GONÇALVES (1998), as quais foram identificadas de acordo com as características descritas por KEITH (1953). De cada grupo experimental, coletaram-se e analisaram-se cem larvas de terceiro estádio (L3) obtidas das culturas de cada grupo estabelecido em cada propriedade.

As médias aritméticas do número de ovos nas fezes, para cada grupo tratado (OPGt), foram calculadas e comparadas com as médias contadas no grupocontrole (OPGc) e a redução da contagem de ovos nas fezes (RCOF) determinada pela fórmula descrita por COLES et al. (2006): 
$\mathrm{RCOF}=[1-($ OPGt $/$ OPGc $)] \times 100$, em que: $\mathrm{RCOF}=$ teste de redução da contagem de ovos por grama de fezes; $\mathrm{OPGt}=$ média do número de ovos por grama de fezes do grupo de animais tratados; $\mathrm{OPGc}=$ média do número de ovos por grama de fezes do grupo-controle.

Os dados obtidos foram analisados pelo programa estatístico RESO (WURSTHORN \& MARTIN, 1990), seguindo-se as instruções WAAVP (COLES et al., 1992), em que a resistência está presente se a percentagem de redução da contagem de ovos for inferior a $95 \%$ e se o limite inferior do intervalo de confiança a $95 \%$ for menor que $90 \%$.

\section{RESULTADOS E DISCUSSÃO}

Ao se comparar o desempenho dos medicamentos ivermectina e albendazole no controle das espécies de endoparasitas específico para caprinos utilizados nas propriedades, de acordo com os critérios estabelecidos pelo programa RESO, a ivermectina apresentou menor resistência que o albendazole. Das trinta propriedades estudadas, na análise do percentual individual de Haemonchus contortus e Trichostrongylus sp., o resultado é como segue: em relação ao $H$. contortus, o percentual de população de helmintos resistentes ao albendazole foi de $90 \%$ nas propriedades estudadas e à ivermectina de apenas 36,6\% (Tabela 1). Trata-se de resultados que se assemelham aos encontrados por MELO et al. (2003).

TABELA 1. Prevalência (\%) de H. contortus em caprinos, resistentes ao albendazole e à ivermectina em propriedades do município de Mossoró, RN, segundo classificações RESO

\begin{tabular}{|c|c|c|}
\hline H. contortus & Classif & RESO \\
\hline Anti-helmínticos & Sensível (\%) & Resistente (\%) \\
\hline Ivermectina & $19(63,4)$ & $11(36,6)$ \\
\hline Albendazole & $3(10,0)$ & $27(90,0)$ \\
\hline
\end{tabular}

O H. contortus foi também o helminto mais prevalente, sendo encontrado em todos os grupos tratados. Resultados similares foram encontrados por VIEIRA \& CAVALCANTE (1999) e BARRETO et al. (2002), no Nordeste brasileiro, e MATTOS et al. (2000), no Rio Grande do Sul. MATTOS et al. (2003), em análise da sensibilidade dos nematodeos gastrintestinais de caprinos à ivermectina, na região da Grande Porto Alegre, verificaram que o $H$. contortus foi o helminto mais prevalente, representando um percentual de $96 \%$ da população encontrada no grupo-controle.

Em relação ao Trichostrongylus sp., a prevalência foi de $70 \%$ nas propriedades estudadas. O percentual de população de helmintos resistentes atingiu, para o tratamento com ivermectina, a resistência de 33,3\% nas propriedades. No tratamento com albendazole, foi de $42,8 \%$ (Tabela 2 ).

TABELA 2. Prevalência (\%) de Trichostrongylus sp. em caprinos, resistentes ao albendazole e à ivermectina em propriedade do município de Mossoró, RN, segundo classificações RESO

\begin{tabular}{|c|c|c|c|}
\hline $\begin{array}{c}\text { Trichostrongylus } \\
\text { sp. }\end{array}$ & Classificação & RESO & $\begin{array}{c}\text { *Não } \\
\text { presentes }\end{array}$ \\
\hline Anti-helmínticos & Sensível (\%) & $\begin{array}{c}\text { Resistente } \\
(\%)\end{array}$ & \\
\hline Ivermectina & $14(66,7)$ & $7(33,3)$ & 9 \\
\hline Albendazole & $12(57,2)$ & $9(42,8)$ & 9 \\
\hline
\end{tabular}

*Propriedades em que não foram encontrados Trichostrongylus sp.

O percentual de helmintos resistentes para os dois princípios ativos é alto e torna-se alarmante, ao se verificar o percentual de nematodeos que possuem resistência ao albendazole: $90 \%$ das propriedades (Tabela 3 ). Resultados semelhantes referem a resistência de nematodeos de caprinos aos benzimidazóis, como obtidos por THEODORIDES et al. (1970), ANDERSEN \& CHISTOFFERSON (1973) e McGREGOR et al. (1980). MELO et al. (2003), em um experimento utilizando 25 criações de ovinos e caprinos localizadas no estado do Ceará, verificaram que $87,5 \%$ delas apresentaram nematodeos resistentes aos benzimidazóis e 37,5\% à ivermectina.

Em ordem de resistência, observou-se que a espécie $H$. contortus foi a que obteve maior índice de resistência, seguida pelos gêneros Trichostrongylus sp. e Oesophagostomun sp. (Tabela 4). Esses resultados estão de acordo com os relatados por MELO et al. (2003), que encontraram Haemonchus sp., Trichostrongylus sp., Ostertagia sp., no Nordeste. Ostertagia sp. não é encontrado no município de Mossoró, RN. 
TABELA 3. Rebanhos caprinos do município de Mossoró, RN, com presença de resistência anti-helmíntica ao albendazole e à ivermectina

\begin{tabular}{lcc}
\hline \multicolumn{2}{c}{ Propriedades } \\
\hline Anti-helmínticos & Sensível(\%) & Resistente(\%) \\
\hline Ivermectina & $17(56,7)$ & $13(43,3)$ \\
Albendazole & $3(10,0)$ & $27(90,0)$ \\
\hline
\end{tabular}

TABELA 4. Prevalência de nematodeos de caprinos resistentes à ivermectina e ao albendazole

\begin{tabular}{lcc}
\hline Nematodeo & \multicolumn{2}{c}{ Droga anti-helmíntica } \\
\hline & Ivermectina & Albendazole \\
\hline & $\mathrm{N}^{\text {o }}$ de proprieda- & $\mathrm{N}^{\mathrm{o}}$ de proprieda- \\
& des $(\%)$ & des $(\%)$ \\
\hline Haemonchus contortus & $11(36,6)$ & $27(90,0)$ \\
Trichostrongylus sp. & $7(33,3)$ & $9(42,8)$ \\
Oesophagostomun $\mathrm{sp}$. & $1(6,6)$ & $3(20,0)$ \\
\hline
\end{tabular}

A redução na contagem de ovos nas fezes (RCOF) após o tratamento com ivermectina e albendazole variou entre $43 \%$ e $100 \%$ e $29 \%$ e $100 \%$, respectivamente (Tabela 5). O percentual de redução diverge do encontrado por MELO et al. (2003). Esses autores, avaliando o percentual de RCOF, obtiveram, como resultado, uma variação de $60 \%$ a $100 \%$ e $2 \%$ a $96 \%$ para ivermectina e benzimidazol, respectivamente. Resultados similares foram relatados por MATTOS et al. (2003), ao testarem a ivermectina na região sul do Brasil. Já BARRETO \& SILVA (1999), em avaliação do RCOF no estado da Bahia para albendazole após dez dias de tratamento, obtiveram a percentagem de 79,31\%. Em Porto Alegre, MATTOS et al. (2004) verificaram a eficácia da ivermectina em $42,10 \%$ aos quatorze dias pós-tratamento. VIEIRA \& CAVALCANTE (1999), em experimento utilizando drogas anti-helmínticas derivadas dos benzimidazóis e imizóis, por meio do RCOF, verificaram que, de 34 rebanhos caprinos no estado do Ceará, 17,6\% dos nematodeos gastrintestinais estavam resistentes ao oxfendazole (derivado do benzimidazol), $20,6 \%$ ao levamizole e $35,3 \%$ apresentaram resistência múltipla a ambos os grupos químicos avaliados.

Os resultados obtidos com a cultura de larvas demonstraram que $100 \%$ das infecções helmínticas de caprinos eram por helmintos da superfamília Trichostrongyloidea e Rhabdiasoidea. Os nematodeos encontrados foram Haemonchus contortus, Strongyloides sp., Trichostrongylus sp. e Oesophagostomun sp. (Tabela 6), o que está de acordo com o descrito por AHID et al. (2008) em avaliação dos parasitos gastrintestinais de caprinos da região oeste do Rio Grande do Norte. A identificação das larvas infectantes mostrou que os nematodeos sobreviventes às medicações testadas foram principalmente Haemonchus contortus, seguido pelo Strongyloides sp. Trata-se de resultados que concordam com os encontrados por AHID et al. (2007), em pesquisas feitas em caprinos da zona da mata em Alagoas.

No presente trabalho, de acordo com as coordenadas geográficas de cada propriedade no município de Mossoró, RN (Tabela 7), nas trinta propriedades marcadas por GPS (Figura 1), houve nematodeos resistentes para o albendazole. A resistência ao medicamento encontra-se largamente distribuída por todo município, concentrando-se na região oeste da cidade (Figura 2).

A resistência à ivermectina está distribuída no município de Mossoró, RN, mas em menor proporção, ao se comparar com o albendazole. A distribuição geográfica das treze propriedades resistentes à ivermectina concentrou-se nas regiões oeste e sudeste da cidade (Figura 3 ).

Em todas as propriedades que continham nematodeos resistentes à ivermectina, 100\% delas estavam resistentes ao albendazole simultaneamente.

A análise dos questionários revelou que os anti-helmínticos mais usados pertenciam aos grupos dos benzimidazóis e avermectinas, e em menor escala dos imidazóis.

Em dezenove propriedades $(63,3 \%)$ os antihelmínticos utilizados pertenciam apenas ao grupo dos benzimidazóis, dez $(33,3 \%)$ à ivermectina e um $(3,3 \%)$ aos imidazóis. Não foi evidenciado uso simultâneo de dois ou mais princípios ativos em uma mesma propriedade. Tais dados discordam de VIEIRA \& CAVALCANTE (1999), em estudo realizdo em rebanhos caprinos no estado do Ceará. Esses autores verificaram que em dez propriedades $(29,4 \%)$ os anti-helmínticos 
utilizados pertenciam apenas ao grupo dos imidazóis $(20,6 \%)$ ou apenas ao dos benzimidazóis $(8,8 \%)$ e que algumas propriedades utilizavam simultaneamente grupos de princípios ativos diferentes.

O perfil epidemiológico das propriedades resistentes no município de Mossoró, $\mathrm{RN}$, revelou que, de acordo com os questionários, os criadores de caprinos adotavam um sistema de manjo animal ineficiente.
Também evidenciou um despreparo e desconhecimento quanto ao uso e dosagem correta dos anti-helmínticos. Em diversas ocasiões, realizaram-se subdosagem e superdosagem dos medicamentos. Os proprietários vermifugavam todo o rebanho sem respeitar um intervalo considerável de dias entre uma administração e outra. Evidenciou-se rápida rotação de grupos de princípios ativos em curtos intervalos de tempo.

TABELA 5. Redução na contagem de ovos nas fezes (RCOF) de caprinos tratados com ivermectina e albendazole após dez dias pósvermifugação

\begin{tabular}{|c|c|c|c|c|c|}
\hline \multirow[t]{2}{*}{ Propriedade } & \multirow{2}{*}{$\begin{array}{c}\text { Controle } \\
\text { OPG médio* }\end{array}$} & \multicolumn{2}{|c|}{ Ivermectina } & \multicolumn{2}{|c|}{ Albendazole } \\
\hline & & OPG médio* & RCOF (\%) & OPG médio* & $\mathrm{RCOF}(\%)$ \\
\hline 1 & 910 & 120 & 87 & 180 & 80 \\
\hline 2 & 1.740 & 150 & 91 & 360 & 79 \\
\hline 3 & 650 & 50 & 92 & 70 & 89 \\
\hline 4 & 520 & 0 & 100 & 30 & 94 \\
\hline 5 & 920 & 20 & 98 & 250 & 73 \\
\hline 6 & 830 & 130 & 84 & 310 & 63 \\
\hline 7 & 1.660 & 630 & 62 & 700 & 58 \\
\hline 8 & 2.210 & 40 & 98 & 630 & 91 \\
\hline 9 & 850 & 110 & 87 & 190 & 78 \\
\hline 10 & 1.210 & 520 & 57 & 300 & 75 \\
\hline 11 & 2.150 & 110 & 95 & 940 & 56 \\
\hline 12 & 2.330 & 230 & 90 & 940 & 60 \\
\hline 13 & 1.542 & 25 & 98 & 417 & 73 \\
\hline 14 & 2.100 & 30 & 99 & 1.110 & 47 \\
\hline 15 & 700 & 100 & 86 & 220 & 69 \\
\hline 16 & 717 & 408 & 43 & 292 & 59 \\
\hline 17 & 785 & 123 & 84 & 292 & 63 \\
\hline 18 & 709 & 309 & 56 & 345 & 51 \\
\hline 19 & 564 & 45 & 92 & 400 & 29 \\
\hline 20 & 520 & 0 & 100 & 30 & 94 \\
\hline 21 & 900 & 0 & 100 & 464 & 48 \\
\hline 22 & 610 & 0 & 100 & 230 & 62 \\
\hline 23 & 330 & 0 & 100 & 40 & 88 \\
\hline 24 & 690 & 0 & 100 & 10 & 99 \\
\hline 25 & 720 & 0 & 100 & 190 & 74 \\
\hline 26 & 550 & 0 & 100 & 60 & 89 \\
\hline 27 & 733 & 13 & 98 & 73 & 90 \\
\hline 28 & 1.080 & 20 & 98 & 400 & 63 \\
\hline 29 & 575 & 8 & 99 & 25 & 96 \\
\hline 30 & 2.980 & 10 & 100 & 0 & 100 \\
\hline
\end{tabular}

* Média aritmética do número de ovos por grama de fezes dos animais de cada grupo. 
TABELA 6. Composição da cultura de larvas, após dez dias da administração dos tratamentos, em caprinos nas propriedades estudadas

\begin{tabular}{|c|c|c|c|c|c|c|c|c|c|c|c|c|}
\hline \multirow{3}{*}{ Propriedades } & \multicolumn{12}{|c|}{ Tratamentos } \\
\hline & \multicolumn{4}{|c|}{ Controle $(\%)$} & \multicolumn{4}{|c|}{ Ivermectina (\%) } & \multicolumn{4}{|c|}{ Albendazole (\%) } \\
\hline & $H$. & $T$. & $O$ & $S$ & $H$ & $T$. & $O$ & $S$ & $H$. & $T$. & $O$ & $S$ \\
\hline 1 & 28 & 0 & 4 & 68 & 10 & 0 & 0 & 90 & 35 & 0 & 1 & 64 \\
\hline 2 & 43 & 0 & 25 & 32 & 9 & 1 & 0 & 90 & 36 & 1 & 0 & 63 \\
\hline 3 & 44 & 22 & 0 & 34 & 55 & 45 & 0 & 0 & 37 & 11 & 0 & 52 \\
\hline 4 & 12 & 2 & 0 & 86 & 2 & 0 & 0 & 98 & 20 & 0 & 0 & 80 \\
\hline 5 & 60 & 1 & 5 & 34 & 30 & 0 & 0 & 70 & 45 & 0 & 0 & 55 \\
\hline 6 & 92 & 2 & 6 & 0 & 31 & 68 & 0 & 1 & 91 & 7 & 1 & 1 \\
\hline 7 & 13 & 4 & 15 & 68 & 78 & 0 & 0 & 22 & 41 & 2 & 5 & 52 \\
\hline 8 & 10 & 3 & 0 & 87 & 92 & 8 & 0 & 0 & 24 & 5 & 0 & 71 \\
\hline 9 & 50 & 2 & 2 & 46 & 78 & 1 & 0 & 21 & 58 & 0 & 0 & 42 \\
\hline 10 & 59 & 9 & 2 & 30 & 80 & 12 & 2 & 6 & 60 & 0 & 0 & 40 \\
\hline 11 & 57 & 2 & 0 & 41 & 51 & 1 & 0 & 38 & 59 & 0 & 0 & 41 \\
\hline 12 & 58 & 1 & 0 & 41 & 74 & 1 & 0 & 25 & 59 & 0 & 0 & 41 \\
\hline 13 & 66 & 2 & 6 & 26 & 45 & 2 & 0 & 53 & 35 & 0 & 0 & 65 \\
\hline 14 & 38 & 1 & 6 & 55 & 17 & 0 & 0 & 83 & 41 & 1 & 2 & 52 \\
\hline 15 & 79 & 5 & 0 & 16 & 90 & 2 & 0 & 90 & 97 & 3 & 0 & 0 \\
\hline 16 & 16 & 0 & 0 & 84 & 24 & 1 & 0 & 75 & 16 & 4 & 0 & 80 \\
\hline 17 & 55 & 0 & 0 & 45 & 100 & 0 & 0 & 0 & 35 & 1 & 0 & 64 \\
\hline 18 & 24 & 2 & 0 & 74 & 14 & 0 & 0 & 86 & 36 & 6 & 0 & 58 \\
\hline 19 & 55 & 7 & 1 & 37 & 56 & 4 & 0 & 40 & 72 & 5 & 8 & 20 \\
\hline 20 & 28 & 0 & 0 & 72 & 2 & 0 & 0 & 98 & 6 & 0 & 0 & 94 \\
\hline 21 & 30 & 0 & 16 & 54 & 20 & 3 & 5 & 72 & 15 & 1 & 4 & 80 \\
\hline 22 & 30 & 0 & 8 & 62 & 38 & 0 & 0 & 62 & 23 & 0 & 0 & 77 \\
\hline 23 & 91 & 0 & 0 & 9 & 1 & 0 & 0 & 99 & 100 & 0 & 0 & 0 \\
\hline 24 & 81 & 2 & 5 & 12 & 20 & 1 & 0 & 79 & 56 & 0 & 0 & 44 \\
\hline 25 & 89 & 11 & 0 & 0 & 99 & 1 & 0 & 0 & 97 & 3 & 0 & 0 \\
\hline 26 & 65 & 10 & 5 & 20 & 71 & 14 & 1 & 4 & 98 & 2 & 0 & 0 \\
\hline 27 & 86 & 14 & 0 & 10 & 30 & 0 & 0 & 70 & 37 & 0 & 0 & 63 \\
\hline 28 & 93 & 7 & 0 & 0 & 100 & 0 & 0 & 0 & 96 & 4 & 0 & 0 \\
\hline 29 & 59 & 1 & 2 & 38 & 62 & 6 & 0 & 32 & 90 & 0 & 0 & 10 \\
\hline 30 & 97 & 0 & 3 & 0 & 94 & 4 & 1 & 1 & 68 & 1 & 1 & 30 \\
\hline
\end{tabular}

$H .=H$. contortus; $T .=$ Trichostrongylus sp.; O. = Oesophagostomum sp.; S.= Strongyloides sp. 
TABELA 7. Coordenadas geográficas via satélite das trinta propriedades visitadas

\begin{tabular}{cccc}
\hline Propriedades & Coordenadas & Propriedades & Coordenadas \\
\hline 1 & S5.39998 W37.22451 & 16 & S5.02743 W37.31698 \\
2 & S5.36225 W37.26400 & 17 & S5.43615 W37.19981 \\
3 & S5.08793 W37.27585 & 18 & S5.22715 W37.38785 \\
4 & S5.21180 W37.44444 & 19 & S4.93007 W37.31671 \\
5 & S5.20653 W37.44746 & 20 & S5.08889 W37.34192 \\
6 & S5.21079 W37.44513 & 21 & S5.11494 W37.15999 \\
7 & S5.20655 W37.44748 & 22 & S5.11547 W37.22690 \\
8 & S5.20725W37.44717 & 23 & S5.35315 W37.20858 \\
9 & S5.20922 W37.44695 & 24 & S5.42394 W37.78753 \\
10 & S5.12480 W37.29022 & 25 & S5.11960 W37.35032 \\
11 & S5.31344W37.16932 & 26 & S5.30678 W37.42174 \\
12 & S5.31518 W37.18233 & 27 & S5.01919 W37.16245 \\
13 & S5.21105W37.44497 & 28 & S5.10854 W37.53341 \\
14 & S5.25670 W37.24236 & 29 & S5.30430 W37.41069 \\
15 & S5.09899 W37.51077 & 30 & S5.48784 W37.76234 \\
\hline
\end{tabular}

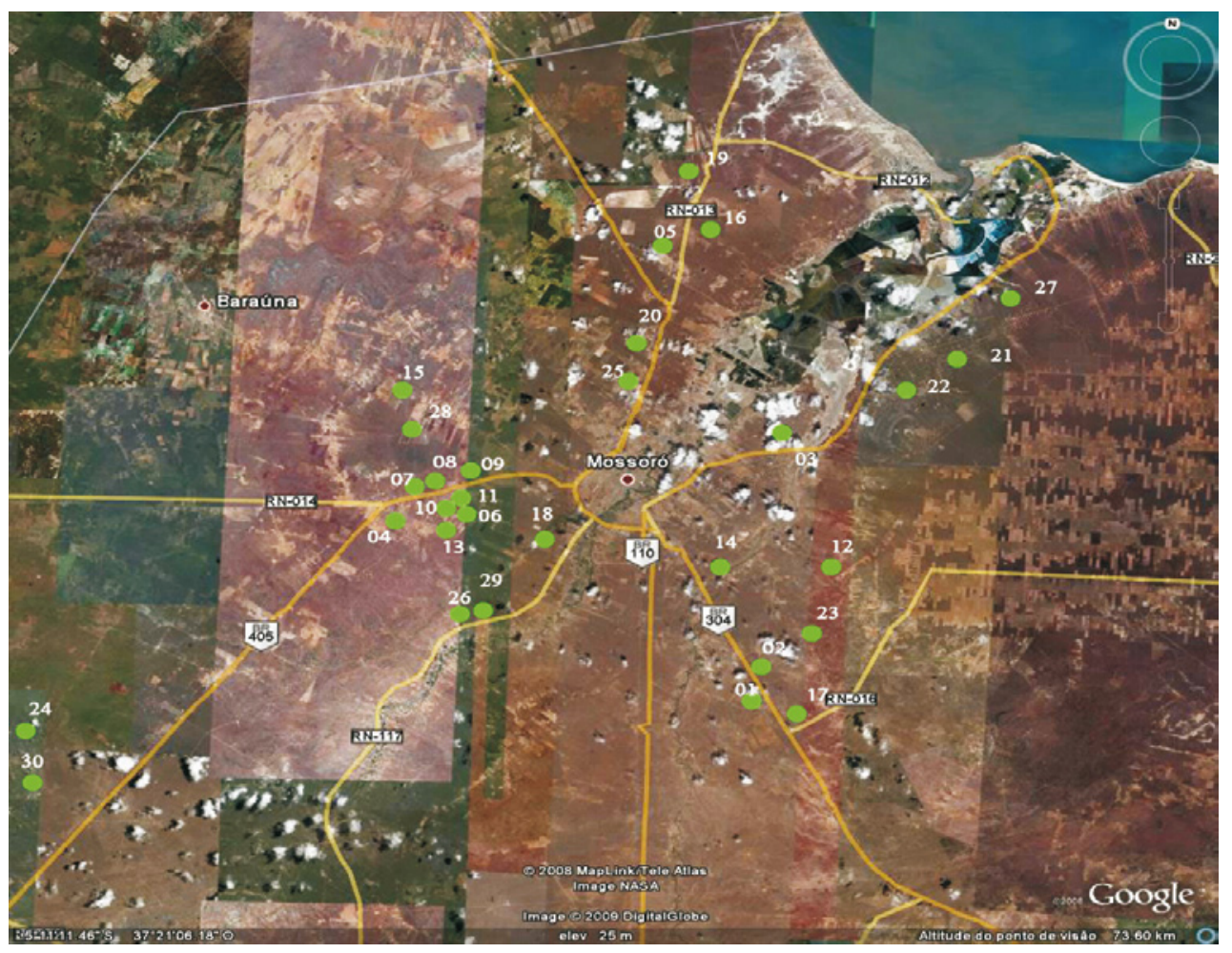

FIGURA 1. Vista aérea do município de Mossoró, RN, na altitude de 73,6 km. Pontos verdes sinalizam as trinta propriedades visitadas. 


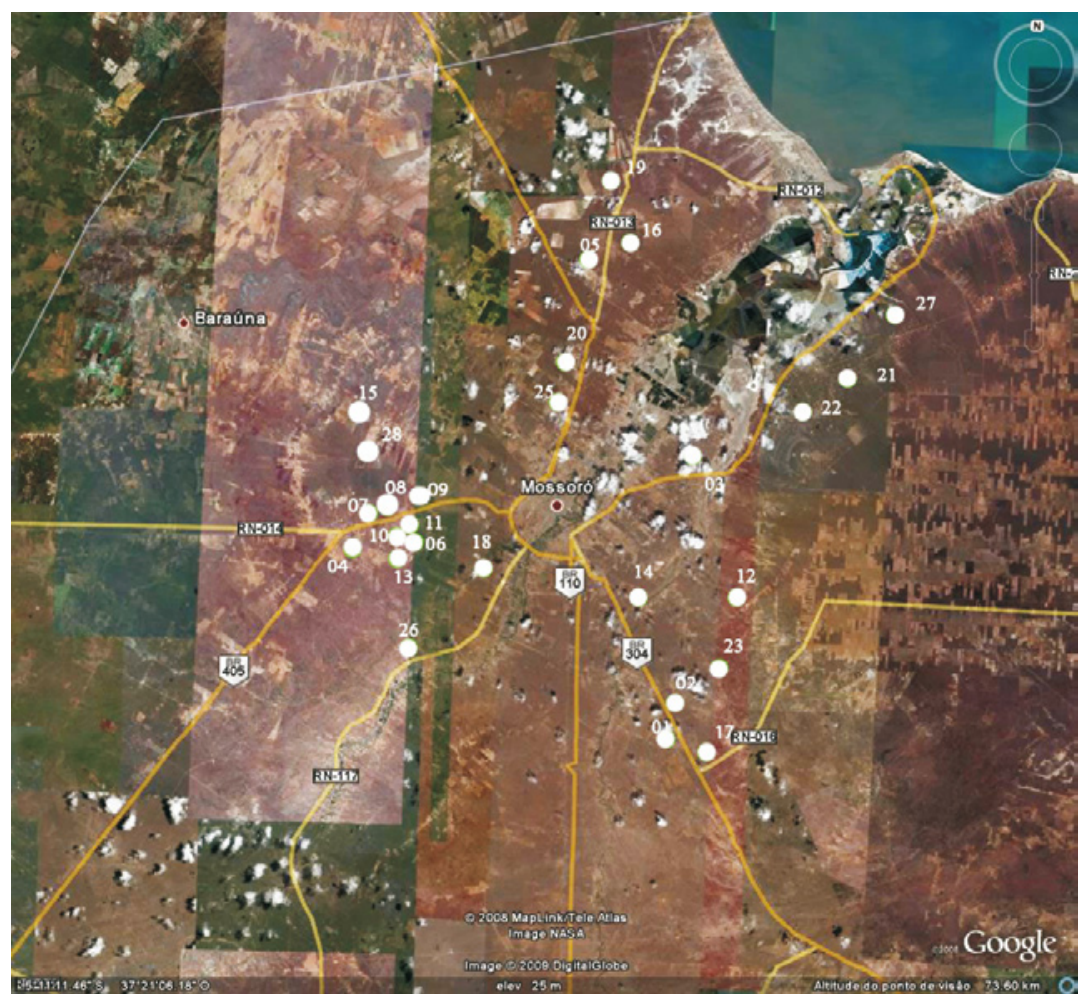

FIGURA 2. Vista aérea do município de Mossoró, RN, na altitude de 73,6 Km. Pontos brancos sinalizam as 27 propriedades resistentes ao albendazole.

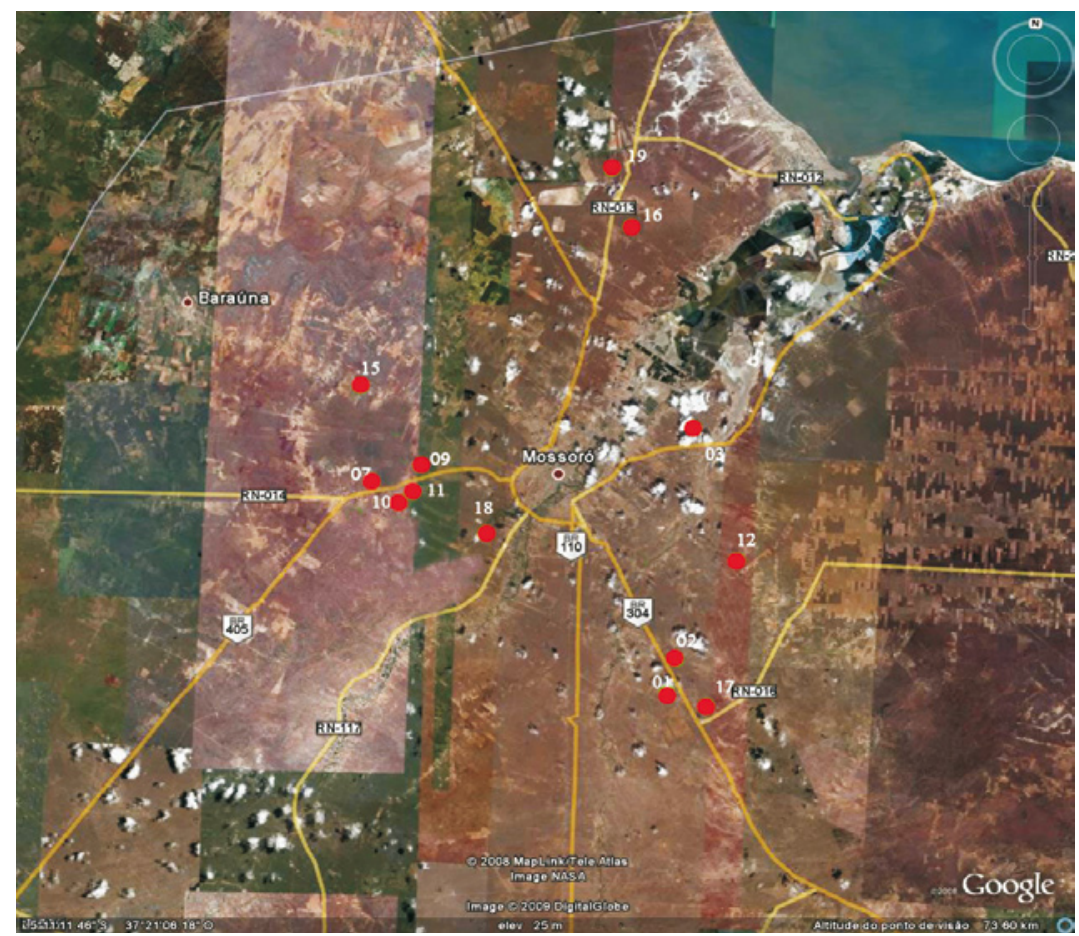

FIGURA 3. Vista aérea do município de Mossoró, RN, na altitude de 73,6 Km. Pontos vermelhos sinalizam as treze propriedades resistentes à ivermectina. 


\section{CONCLUSÃO}

Os resultados obtidos indicam que os nematoides gastrintestinais de caprinos localizados no município de Mossoró, RN, estão resistentes, em sua maioria, ao anti-helmíntico albendazole e em menor escala à ivermectina.

\section{AGRADECIMENTOS}

À Associação dos Caprinos e Ovinos de Mossoró e Região (ASCCOM), à Empresa de Assistência Técnica e Extensão Rural (EMATER), à BIOFARM - Tecnologia em Veterinária e à Embrapa Caprinos.

\section{REFERÊNCIAS}

ACOSC. Caprinovinocultura é um bom negócio para o pequeno produtor. Disponível em $:<$ http://www.acosc.org.br $/>$. Acesso em: 2 nov. 2006.

AHID, S. M. M.; CAVAlCANTE, M. D. A.; BEZERRA, A. C. D. S.; SOARES, H. T. S.; PEREIRA ,R. H. M. A. Eficácia antihelmíntica em rebanho caprino no Estado de Alagoas, Brasil. Acta Veterinária Brasílica, v. 1, n. 2, p. 56-59, 2007.

AHID, S. M. M.; SUASSUNA, A. C. D.; MAIA, M. B.; COSTA, V. M. M.; SOARES, H. S. Parasitos gastrintestinais em caprinos e ovinos da região Oeste do Rio Grande do Norte, Brasil. Ciência Animal Brasileira, v. 9, n. 1, p. 212-218, 2008.

ALBERTI, H.; ALBERTI A. L. L.; BORDIN, B. E. L.; SILVA JR., C. I. F. Algumas considerações sobre a resistência dos parasitos aos antiparasitários e métodos de avaliação. A Hora Veterinária, v. 21, n. 123 , p. 36-40, 2001.

ANDERSEN, F. L.; CHRISTOFERSON P. V. Efficacy of haloxon and thiabendazole against gastrointestinal nematodes in sheep and goats in the Edwards Pleteau area of Texas. Journal Veterinary, v. 34, n. 11, p. 1395-1398, 1973.

BARRETO, M. A.; ALMEIDA, M.A. O.; SILVA, A.; REBOUÇAS, I.; MENDONÇA, L. R. Resistência anti-helmíntica em rebanhos caprinos no estado da Bahia. In: CONGRESSO BRASILEIRO DE MEDICINA VETERINÁRIA(CONBRAVET), 29., 2002, Anais... Gramado: SBMV/SOVERGS, 2002. Disponível em: < http://www. conbravet.com.br/>. Acesso em: 22 jun. 2002.

BARRETO, M. A.; SILVA, J. S. Avaliação da resistência de nematodeos gastrintestinais em rebanhos caprinos do Estado da Bahia: resultados preliminares. In: SEMINÁRIO BRASILEIRO DE PARASITOLOGIA VETERINÁRIA, 1999, Salvador, BA.
Anais... Salvador: Colégio Brasileiro de Parasitologia Veterinária, 1999, 160 p. Disponível em: <http://www.cnpc.embrapa.br/>. Acesso em: 15 dez. 1999.

CHARLES, T. P.; POMPEU, J.; MIRANDA, D. B. Efficacy of three broad-spectrum anthelmintics against gastrointestinal nematode infections of goats. Veterinary Parasitology, v. 34, p. 71-75, 1989.

COLES, G. C.; JACKSON, F.; POMROY, W. E.; PRICHARD R. K.; SON-HIMMELSTJERNA, G. V.; SILVESTRE, A.; TAYLOR, M. A.; VERCRUYSSE, J. The detection of anthelmintic resistance in nematodes of veterinary importance. Veterinary Parasitology, v. 136, p. 167-185, 2006.

COLES, G. C.; BAUER, C.; BORGSTEEDE, F. H. M.; GEERTS, S.; KLEI, T. R.; TAYLOR, M. A.. World association for the advancement of veterinary parasitology (WAAVP) methods for the detection of anthelmintic resistance in nematodes of veterinary importance. Veterinary Parasitology, v. 44, p. 35-44, 1992.

COSTA JÚNIOR, G, S.; MENDONÇA, I. L.; CAMPELO, J. E. G.; CAVALCANTE, R. R.; FILHO, L. A. D.; NASCIMENTO, I. M. R.; ALMEIDA, E. C. S.; CHAVES, R. M. Efeito de vermifugação estratégica, com princípio ativo à base de ivermectina na incidência de parasitos gastrintestinais no rebanho caprino da UFPI. Ciência Animal Brasileira, v. 6, n. 4, p. 279-286, 2005.

GORDON, H. M.; WHITLOCK, H. V. Anew technique for couting nematode eggs in sheep faeces. Journal of the Commonwealth Scientific and Industrial Research Organization, v. 12, p. 50-52, 1939.

IBGE. 2003. Disponível em : < http://www.ibge.gov.br >. Acesso em: 3 dez. 2003.

KEITH, R. K. The differential of the infective larval of some common nematode parasites of cattle. Australian Journal Zoollogy, v. 2, p. 223-230, 1953.

KERBOUEF, D.; HUBERT, J. Benzimidazole resistance of field strains of nematodes from goats in France. Veterinary Record, v. 116, n. $5,1985,133$ p.

KETTLE, P. R.; VLASSOFF A.; REID, T. C; HORTON, C. T. A survey of nematode control of measure used by milking goats farmers and of anthelmintic resistance on their farms. Journal of Zoology Veterinary, v. 31, n. 8, p. 139-143, 1983.

MATTOS , M. J. T.; SCHMIDT, V; BASTOS, C. D. Atividade ovicida de dois fármacos em caprinos naturalmente parasitados por nematodeos gastrintestinais, RS, Brasil. Ciência Rural, v. 30, n. 5 , p. $893-895,2000$.

MATTOS M. J. T.; OLIVEIRAC. M. B.; GOUVÊAA. S.;ANDRADE C. B. Haemonchus resistente à lactona macrocíclica em caprinos naturalmente parasitados. Ciência Rural, v. 34, n. 3, p. 879-883, 2004. 
MATTOS, M. J. T.; CASTRO, E. S. Utilização de anti-helmínticos no controle de verminose caprina no Estado do Rio Grande do Sul. A Hora Veterinária, v. 22, n. 130, p. 52-54, 2002.

MATTOS, M. J. T.; OLIVEIRA, C. M. B.; GOUVEA, A. S.; ANDRADE, C. B. Sensibilidade dos nematodeos gastrintestinais de caprinos ao ivermectin na região da Grande Porto Alegre, RS. Acta Scientiae Veterinariae, v. 31, n. 3, p. 155-160, 2003.

MCGREGOR, B. A.; ADOLPH A. J.; CAMPBEL, N. J. Occurrence of anthelmintic resistance in goats in Victoria. Australian Animal, p. 13-159, 1980.

MELO A. C. F. L.; BEVILAQUA C. M. L.; VILLAROEL A. S.; GIRÃO M. D. Resistência a anti-helmínticos em nematoides gastrintestinais de ovinos e caprinos, no município de Pentecoste, Estado do Ceará. Ciência Animal, v. 8, p. 7-11, 1998.

MELO, A. C. F. L.; REIS, I. F.; BEVILAQUA, C. M. L.; VIEIRA, L. S.; ECHEVARRIA, F. A. M.; MELO, L. M. Nematodeos resistentes a anti-helmínticos em rebanhos de ovinos e caprinos do estado do Ceará, Brasil. Ciência Rural, v. 33, p. 339-344, 2003.

MOLENTO, M. B. Resistência de helmintos em ovinos e caprinos. Revista Brasileira de Parasitologia Veterinária, v. 13, p.82-85, 2004.

PAIVA, F.; SATO, M. O.; ACUÑA, A. H.; JENSEN, J. R.; BRESSAN, M. C. R. V. Resistênciaà ivermectina constatada em Haemonchus placei e Cooperia punctata em bovinos. Hora veterinária. Disponível em:< http://www.veterinary-associates.com />. Acesso em: 25 fev. 2007.
SANGSTER, N. C. Managing parasiticide resistance. Veterinary Parasitology, v. 98, p. 89-109, 2001.

SIMPLÍCIO, A. A. Caprino-ovinocultura: uma alternativa à geração de emprego e renda. Disponível em: < http://www.cnpc. embrapa.br/artigo-6.htm >. Acesso em: 3 nov. 2006.

THEODORIDES V. J.; SCOTT, G. C.; LADERMAN, M. Efficacy of parbendazole against gastrointestinal nematodes in goats. Jornal of Veterinary, v. 31, n. 5, p. 857-863, 1970.

UENO, H.; GONÇALVES, P. C. Manual para diagnóstico das helmintoses de ruminantes. 4. ed. Tokyo: Japan International Cooperation, 1998, 143 p.

VIEIRA, L. S. Atividade ovicida in vitro e in vivo dos benzimidazóis: oxfendazole, fenbendazole, albendazole e thiabendazole em nematodeos gastrintestinais de caprinos. 1986. 115 f. Tese, (Mestrado em Ciências Veterinárias) - Departamento de Ciências animais, Universidade Federal do Rio Grande do Sul, Porto Alegre, 1986. Disponível em: < http://www.cnpc.embrapa.br/dissertacao. htm $>$. Acesso em: 26 jun. 2009.

VIEIRA, L. S.; CAVALCANTE, A. C. R. Resistência antihelmíntica em rebanhos caprinos no Estado do Ceará. Pesquisa Veterinária Brasileira, v. 19, n. 3-4, p. 99-103, 1999.

WURSTHORN, L.; MARTIN, P. Reso: faecal egg count reduction test (FECRT) Analysis Program. 2.01. Parkville: CSIRO Animal Health Research Laboratory, 1990. 